\title{
Buchbesprechungen
}

Friedrich Müller, Recht - Sprache - Gewalt. Elemente einer Verfassungscheorie I, Berlin 1975, 43 S., DM 12,60, (I); ders., Juristische Methodik und Politisches System. Elemente einer Verfassungstbeorie II, Berlin 1976, 127 S., DM 36,-, (II); ders., Die Einheit der Verfassung. Elemente einer Verfassungsibeorie III, Berlin 1979, 268 S., DM 68,-, (III); ders., Juriscische Mechodik, 2. Auf., Berlin 1976, 327 S., DM 48,-, (IV); alle Bände bei Duncker und Humbloc

Das wissenschafeliche Werk des Heidelberger Staatsrechulers Friedrich Müller läßt sich - blender man die verfassungsgeschichulicher und rechesphilosophischen Arbeiten aus' - grob einteilen in die drei Themenbereiche Kritik der Rechtswissenschaft und Rechespraxis, juristische Methodik und Verfassungstheorie. Den leichtesten Zugang zu ejnem Werk, dessen Problemfülle und Sprache hohe Anforderungen an den Verscehensprozeß stcllen, eröfínet die Rechtskritik. Unter diesem Aspekt ist Müllors neuestes Buch "Die Einheir dex Verfassung « denn auch jüngst von einem Verereter der staatssechtljchen Linken rezipiert worden.'

Die Schrif belegr an der Geschichte der bundesdeusschen Verfassungsrechtsprechung die unrerschiedliche Verwendung des Arguments von der wEinheit der Verfassung" durch die beiden Senate des BVeriG. Im Südweststaats-Urceil (BVerfGE I, $x_{4} \mathrm{ff}$. ) an-

1 Zu nernen wären hier: ders., Korporation und Assoziacion. Eine Problemgeschicbte der Vereinigungsfreiheit in dcutschen Vormärz, Bcrlin r 965, und ders, Endremdung. Zur anchro pologischen Begrindung der Staatstheorie bei Rousseav, Hegel, Marx, Berbin 1970.

2 Scifert, Haus oder Forum. Wensystem oder olfene Verfassung, in: J. Habcruas (Krsg.), Stichwore zur sGeistigen Situation der Zeir $\%$, Bd. 1, Franktur 1979, S. 121 II. gizipiert der zweite Senat mit den Topoi von der sinneren Einheit $x$ und dem "Sinnzusammenhang « des Grundgesetzes, aus denen die das positive Verfassungsrecht überlagernden "Grundentscheidungen $\propto$ abzuleiten seien, jene Rechesprechungspraxis, die im Namen einer quasi naturrechulichen W/erteordnung die posivive Verfassungsordnung hierarchisch absruft mit dem zweifelhatten Ergebris, daß whöherrangige Wertentscheidungen vorgeblich niederrangige Normen verdrängen. Der erste Senat hingegen bezieht aus der "Einheit der Verfassung* diss Posnlat der Gleichrangigkeit aller Verfassungsnormen und verwirft die These von den nverfassungswióngen Verfassungsnormen $\alpha$. Einig sind sich beidc Senate in der Funkcionsbescimmung des Topas. Die Rede von eincr "Einheit" erspart die sonsı nörigen dogmarischen und methodischen Eiozelschrite in der Rechtsfindung und eignet sich hervorragend zur Produktion ndiffuser, künftig vielseitig einsetzbarer Legirimië̌ä (III, 47). Die in dieser Rechesprechung encwickelte holistische, d. h. in Ganzheiten denkende Argumentation hat Tradivion. In den ancipositiviseisehen Staatslehren der Weimarer Republik wird mic der $*$ Einheit der Verfassung a oder "Einheit des Stares der Kompromißcharakser der Reichsverfassung geleugnet. Smend läßt reale gesellschafuliche Widerspruiche aufgehen in der „Totaliár des Staatesa und der "Totalität seines Integracionsprozesses $*$, Einheit steht gegen eine pluralistische Gesellschafr, auch gegen die Ausstatung bisher unterprivilegierter sozialer Schichten mit gleichen Rechten. Carl Schmirs positiver Verfassungsbegriff, dem die Verfassung dic einmalige Grundentscheidung des deutschen Volkes über wForn und Arr der policischen Einheit ist, reduziert die Verfassung auf ein Substrat bürgerlichrechtsstaadicher Organisauionsprinzipien und privater Freiheitsrechte, während die po- 
litischen Freiheitssechte und die sozialen Grundrechce als bloße Verfassungsgesezze niederen Rang einnehmen. Für Müller verbirge sich hinter dem Konzepc einer Einheic der Verfassung das immer gleiche Bestreben: die Hoffnung auf Einheir in einer antagonistischen Gesellschafr, die Vermeidung offener Konflikte, das Eroveckenwollen des Glaubens an Einheit und innere Harmonie des Ganzen, also die Erzeugung von Legitimität in einer Gesellschaft, die sozial keine ejnheitiche ist und in der einheitliche Wertvorstellungen längst zersroben sind. (III, 232) Gewiß ist die Rede von der Einheit Rhetorik, auch verrät sie ein illusionäres Welıbild, dennoch bleibr sie gefährlich, weil an die Stelle konsistenter Begrünơung juristischer Entscheidung Ideologie tritt.

Seifer hat an dem Buch den ngroßen Scharfsinn in der Destruktion eines von Rechtsprechung und Lehre errichreten halbcheologischen Gebäudesx gelobn zugleich aber ge rügt, der Verfasser verzichte win seiner Kritik - resignierend - auf die explizite Darlegung dex Gegenposition a und lasse diese nur $*$ als Negativ seiner Kricik erkennen*.' Ich kann mir dieses Urreil nur so erkJären, daß der Kriviker das Buch mir einem ausschließlichen Inceresse an den ideologiekritischen Passagen gelesen hat. Müllers Untersuchung der be denklichen Geschichte eines Begriffs, also - um bei der vorgeschlagenen Einueilung zu bleiben - die Arbeit der Rechtskritik, verwendet eir methodisches Instrumentarium, das Auskunt gibe über die verfassungspolitische Option des Aucors. Freilich wird sowohl das Spezifische dieser Methodik als auch die Verfassungscheorie sowie die Vermirtlung beider deutlicher, wenn die vorangegangenen Schrifuer, auf derer inhaldliche Fortführung der Untercitel des Buches $*$ Elemente einer Verfassungstheorie III * verweist. mitberücksichugt werden. Der Ansarz Müllers verbietet die Trennung des Encwurts einer Methodik von der Verfassungstheorie; das Interesse an einer möglichst klaren Darstellung einer komplexen Theorie mag als Entschuldigung dienen, daß im Jolgenden die Trennung crotzdem durchgeführe wird.

Fricdricb Müllers Earwurf eines juriscischen Methodik bewegr sich um die Problempunkte, die auch die Diskussion innerbalb der staatsrechulichen Linken bestimmen. Es gehr um den politischen Stellenwen eincr Metho-

3 A. 2. O., S. 332. dik, die zwei Ansprüchen gieichermaßen genügen soll. Der Ruf nach der Integration der Sozjalwissenschaften in die Rechtswissenschajt und -praxis ist heute zwar nicht mehr so laut wie 1968 in Loccumer Memorandum zur Reform der juristischen Ausbildung, immerhin wird der Anspruch aufrechterhalten, die "Krivische Jusciz * beispielswcise vertritu ihn nach wie vor. Der zweite Anspruch ist ersz später hinzugetreten. Der Radikalen-Er$\mathrm{l}_{\mathrm{a}} \mathrm{B}$ und dic Judikate zur beamtenrechtlichen Gewäurbieteformel haben in der westdeutschen Linken das Bewußrsein für die Bedeurung der gesetzesstaadichen Legalitär sensibilisiert. Gegen die Denunziation der Legalität als substanziosen Gesetzesmechanismus und die Berulung auf eine überpositive und vordemokrazische Legitimitäs harte Ulrich $\mathrm{K}$. Preuß schon 1972 aufgefordert zum Kampf für eine "demokratische Legalitär «." Mit der Rückbesinnung auf den freiheirsverbürgendea und Rechtssicherheit gewährenden Formalcharakter des Rechts ging einher die Wiederentdeckung zunächst der den Wortaut der Verfassung ernsmehmenden Verfassungsintepretation Abendraths' sowie Seiferts Programm des $*$ Kamples um Verfassungspositionen ${ }^{6}$ und sodann die Rehabilirerung des sraatsrechrlichen Posicivismus. Inzwischen hat der Rekurs auf den Verfassungspositivismus bereits die Frage aufgeworfen, ob die Dominanz verfassungsrechulicher Argumentationen in der Linken nicht

4 Preuß, in: Blärer für deuesche und internario nale Politik, 1972, S. 146

s Abendroth, Das Grundgeserz. Eine Einführung in seine polizischen Probleme, 6. Aufl, Pfullingen 1976. Abendrouhs Verfassungsinzesprecavion läfi sich zusammenfassen in den zwei methodischen Srichworten Wordaut und bisorische Auslegung. Dic Entscheidungen dcs Grundgesezzes seien zu erminelo durch die -philologiscb genaue Interpretation der Gescragebungsmaterialien uncer Einbeziehung der politisch-historischen Analyse der Enistehingszeir des Grundgeserzes; oberstes Gcbor aber sei die suriktc Orientierung am Worklaut der Verfassung und damic verbunden die Absage an alle Versuche, von der jeweils akruellen pesellschafdichen Realität, det -Verfassungswirklichkeito aus den Inhalt der Verfassungsgesecze zu bescimmen.

6 Seifert, Kampl um Verfassungsposivionen. FrankưurdMain 1974, S. 105 11. Gemäß diesem juriscischen und politischen Programm sind Verfassungsgesezze soziale Vaffensillstandsbedingungen, die von der Arbeiterklasse immer Deu gegen Restaurationsbestrebungen zu verteidigen scien. 
hinderlich sein könnze bei der Erarbeitung politischer Strategien.' Jedeníalls behandelt die staarsrechtiche Diskussion den Problemkreis, wie eine rechtsstaadichen Prinzipien genügende juristische Methodik in Einklang zu bringen sei mit dern unbestreitbaren Fakturn, daß̉ juriscisches Arbeiten ohne Sozialwissenschaft blinde Praxis bleibe. Daß mit der Wiederentdeckung des Verfassungspositivismus kein "Zurück zu Kelsen gemeint ist, belegt Helmut Ridder, der den Angriffen aut den vielgeschmähren juriscischen Positivismus, dem sich die Bereitschaft eigne, „Normtexte zunächst einmal hinzunehmen und niche von vornherein verfälschen zu wollenж, eire Spitze abbrechen will, zugleich aber aufruth, dem Positivismus den .Star der partiellen historischen, politischen und soziologischen Blindheit zu stechen. ${ }^{8}$

Müllers Arbeiten wollen beiden Anspruichen gerechr werden. Der gesetzespositivisuische Anspruch auf Methodenklarheit und Berechenbarkeit ist dem Entwurf Verpflichrung, die Mängel der pasitivistischen Trennung von Sein und Soilen sind ihm Gewißheit. - Der Gesetzespositivismus kann nichr zul der Seite des bewrußcen Einbeziehens der sozialen Wirklichkeit überwunden werden, wenn man auf der Seite seiner rechtssmarlichen Technizität und Formqualitäten verfassungsgeschichtlich hinter ihn zuriickfällt. Eine nachpositivisusche Rechtsaormeheorie und Mechodik muß versuchen, beiden Varianten von Racionalität gerecht zu werden. . (IV, 27 r) Die Racionalizät juristiscber Methodik erweist sich in der wissenschaftsprakrischen Technik regulärer und incersubjektiv akzepuerbarer Rückführung von Entscheidungsnormen auf Rechrsnormen. Zwei Anforderungen muß diese Technik genügen: sie muß imstande sein, der bürgerlichen Wirtschates- und Verkehrsgesellschaft Berechenbarkeic, Durchschaubarkeit und Regularität der Entscheidungsfindung zu garancieren, und sie muß legitimierend wirken in dem

$7 \mathrm{Vgl}$. Thomas Blanke, Repression und Verfassung: Aspekte zur Funkrioo verfassungsrechtlicher Argumente in politischen Auseinandersezzungen, in: Abendroth, Blanke u. a., Der Kampf um das Grundgeserz. Uber die polizische Bedeutung der Verfassungsinterpretation. Frankhur/Main 1977 , S. If ff.

8 Ridder, Die soziale Ordnuog des Grundgeserzes. Leifaden zu den Grundrechren einer demokravischen Vertassung, Opladen 1978, S. 16, ir.

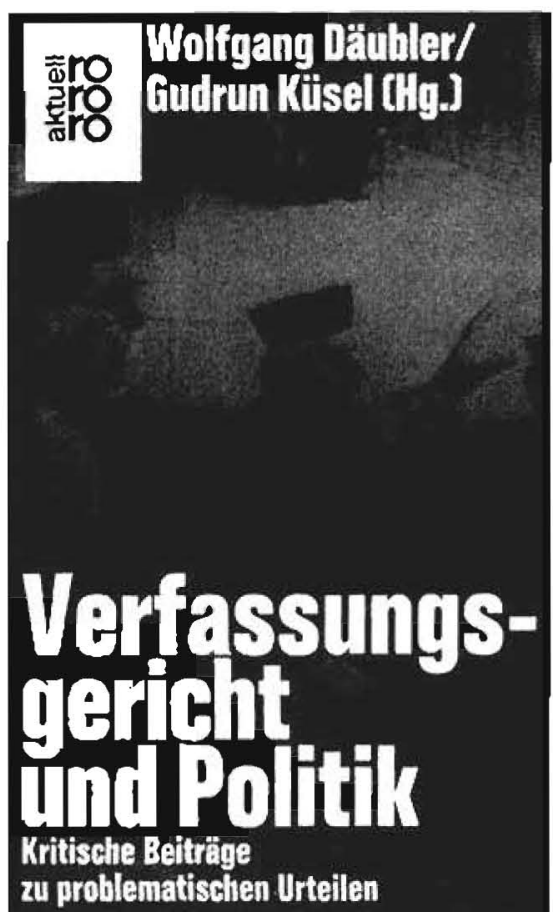

Wolfgang Dăubler/

Gudrun Kilsel ( $\mathrm{Hg}$.)

Vertassungsgericht und Politik

Kritische Beitrăge zu

problematischen Urteilen.

rororo aktuell 4439/DM 7,80

\section{Wolfgang Däubler}

Das Aiteitsrecht

Von der Kinderarbeit zur

Betriebsverfassung. Ein

Leitfaden für Arbeitnehmer.

rororo aktuell 4057/DM 9,80

\section{Wolfgang Däubler}

Das Atheitsrecht 2

Arbeitsplatz - Arbeits-

losigkeit - Kündigung -

Arbeitsgerichtsbarkeit. Ein

Leitfaden für Arbeitnehmer.

rororo aktuell 4275/DM 10,80

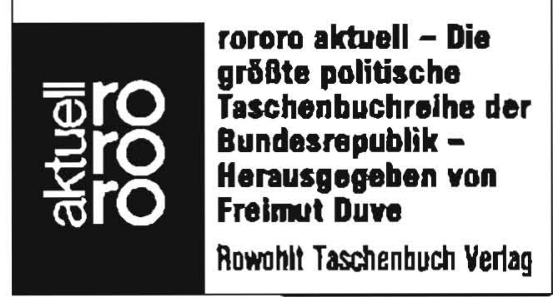


Sinne, daß die Transparenz der Enrschejdungsprozesse oder zumindest der Entscheidungsgründe Kritik und Kontrolle erlaubr und damir der Arspruch des liberalen Verfassungsstaates erfüllbbar bleibr, den status quo und seine Veränderung auf Zuscimmung. Kompromiß und akzepwerbare Mehrheitsmeinung zu stützen. $(1,27)$

Entwickelt wird die juristische Methodik im Medium der Kritik an konkretem, von Rechtsfunktionärer produziertem Material. Dabei zeigt der Entwurt sich sperrig gegenüber der Versuchung, angesichts komplexer Problemkonstellacionen in globale Theorieansärze auszuweichen. Der Rede von der Ineegracion von Rechtswissenschaft und Sozialwissenschaften wird die Frage nach der Incegracionsfähigkeir diescr Wissenschatzen, $d$. $h$. nach dem Siand der deutschen Forschungsund Praxisgeschichee konfrontien. Für die Rechtswissenschaft und Rechtsanwendung bedeuter dies: sie müssen sich bewußr werden darüber, was sie alltäglich cun. Juriscische Selbstreflexion darf niche global ansetzen, sie wird ihre Kraft endialten müssen in der Uncersuchung der Arbeitsweisen der Rechrsfunkrionäre. Wenig sinnvoll ist die Fragestellung nach dem Verhältris von Recht und Wirklichkeit, Recht und Poliuk, wenn die juristischen Arbeitsvorgänge unanalysier belassen werden in der $\times$ Allräglichkei ihrer Probleme, Konflikte und Kompromisse, in der Allräglichkeit von Komperenzen, Arbeitsweisen und Kontrollen von Normen, Insciturionen und von der Veränderung beider $\alpha$. (II, 18 )

Am Beispiel der Rechrsprechung des BVerfG skizzier Müller das Dilemma juristischer Arbeitsweise. Dem positivistischen Dogma, dem die Rechtspraxis verbal ihre Treue erklärt, korrespondien real ein unreflekuener und undurchschaubarer Pragmarismus. Mißbrauchsurteile, Güteraboragungstechniken, materiale Gesetzesvorbehalte, Wensysteme licfern die materialen Inthalte, ${ }^{2}$ Dezisionen durch Rechrsverbiegunga und $\triangle$ Dezisionen durch Rechtsunterstellung sind die Verkahren dieser Rechtspraxis. (II, 21) Die Arbeiz an einer rationalen Methodik muß zunächst zur Sprache bringen, was unterhalb des gesetzesposieivisuschen Dogmas real geschieht. Die Rechtsprechung betreibz nicht Normtextauslegung, sondem Normkonkretisierung. Diese Erkenntnis ist das Fundament des Entwurfs der juristischen Methodik, der Versuch, die Normkonkrecisierung zurückzuzwingen in die Fesseln juristisch-merhodischen Argumenvierens, das selbstgesctzse Zicl.

Der Bestimmung des Vorgangs der Konkretisierung liegt zugrunde eine Rechtsnormthcorie, dic in der Rechrsnorm eine "sachbesuimm - normative Richrlinie" und zudem eine "sachbesumm - normacive Grenze zulässiger Konkrctisienung " erblickt. Demzufolge ist Konkrevisierung der wurch die Norm errnächigte, durch sie sachlich angeregre, in wechselndem, aber so gur wie nie vollständigem Grad bestimmte und bezüglich der Zulässigkeit möglicher Entscheidungsalternativen sachlich begrenzte junstische Arbeits- und Entscheidungsvorgang*. (IV, 139) Eine zentrale Voraussetzung des Entwurfs ist in dieser Definition bereits mitgedacht, die Nonn ist nicht identisch mit dem Normtext. Die Norm konstituiert sich aus Nomecxe und Normbereich, Konkresisierung umfaßt zwei in einem einheitlichen Vorgang zusammengelegic Verfahrensschrit18, Normiextasslegung und Normbereichsanalyse. Bevor der Normbereich bestimmt werden kann, ist aus dem Normtext das Normprogramm - vorstcllbar als Rechisbcfeh) - zu erarbeizen. Erst das Normprogramm schneidet aus den Berührungspunk. ten der Norm mit der sozialen Realizät, dem Sachbereich, den für die Normativität mitkonsrituiven Normbereich heraus. Der Normbereich kann gänzlich rechuserzeugt scin (wie bei Vorschriłten über Fristen, Termine, Rechtswegzuweisungen eac.), dann liefert er keine zusätzlichen Informacionen für die Konkretisienung, die hier beschränkr ist aut die Arbeir der Normcextauslegung. Wenn hingegen das Normprogramm aul soziale Sinkeuran (Ehe, Familie, Handelsgcsellschaften, Grundrechre etc.) verweist, gehören Sachgesicbrspunkte des Normbereichs zu den entscheidungserheblichen Daten.

Die das Normprogramm ermittelnde Normtexrauslegung ist demnach methodisch der erste Schritr im Verfahren der Konkrecisienung, sie genießt aber auch - wie wir noch sehen werden - die sachlich-inhaleliche Priorität. Wenn Müller von Auslegung spricht, meins er nicht das geserzesposicivistische Verfahren, aus formalisierten Obersïzen im syllogistischen Schlußverfahren Entscheidungsnormen abzuleiten. Die Entscheidungsnorm ergibt sich nicht aus der Rechtsnorm, sondern sie wird an der Rechtsnorm gemessen und har sich an ihr zu bewähren. Müller selbsr verweist diesen Arsarz in dic 
sachliche Nähe des Falsifikacionsmodells der krizisch-rationalistischen Wissenschaftslehre. Die herkömmlichen canones haben denn auch nur in Ausnahmefällen Bescimmungswirkung, regelmäßig sind sie nur ein Indiz für den zu ermitreinden Sinn der Norm. So wird von der grammatischen, genetischen, historischen, systematischen und teleologischen Auslegung nur gesprochen a)s von methodologischen Elementen praktischer Normkonkretisierung, die ergänze werden können durch weitere Elemente, die unterteilc sind in unmirelbar normbezogene (neben den canones 2. B. die Prinzipien der Verfassungsintcrpretation, dogmatische Elemente) und solche, die nicht unmiuclbar normbezogen sind und nur Hillsfunkcionen haben ( $z$. B. Theorieelemente, lösungstechnische und rechispolitische Elemente).

Die normtextbezogenen Konkretisierungselemente werden geprït und modifizien in der Auseinanderselzung mit den methodischen Positionen der Rechtsprechung und des Schriftrums. In der immanenten Kricik dieser Positionen wird dem Leser vor Augen geführt, welchen Gewinn an Rationalität der methodisch konsistente Einsatz der normtexibezogenen Konkrcrisierungstechniken erbringen kann. Strukturierung nennt Müller diesen Vorgang der methodischen Zähmung der Konkretisierung durch die in eine Rangfolge gebrachten methodologischen Elemenre. Soweit leuchcet der Entwurt ein und macht deutlich, was Müller unter rationaler Entscheidungsfindung in methodischer Veranrwortlichkeic versteht. Dagegen sind dic Aussagen zur Erarbeirung von Sachgesichrspunkten aus dem Normbereich auffällig unverbindlich. Der Entwurf vermag niche mehr anzubieten als die Aussage, die Realdaten der Normbereiche seien empirisch zu ermitteln und mic den primär sprachlichen Konkretjsierungselementen innerhalb des einzelnen Entscheidungsprozesses zu vermirteln. Der Hinweis aul die Autgabe der Juristenausbildung, für die (achliche Kompetenz dieser Arbeit Sorge zu tragen, und die Forderung nach einer hunktionalen Arbeitsteilung $\mathrm{zwi-}$ schen Juristen und Sozialwissenschatulem könnten zu dem Schluß verleiten, Müller delegicre die Normbereichsanalyse an eine sozialwissenschaftlich fundierte Juristenausbildung und/oder an die Kollegen der sozialwissenschaftlichen Fakulüt. Zwei umfangreiche Entscheidungsanalysen in der oJuristischen Methodik" scheinen den Verdache zu bestäugen. In ciner Analyse wird auf Realdaten überhaupt nicht eingegangen, da der Normbereich in Gänze rechoserzeugr ist, in der anderen Besprechung werden Normbereichselemente nur beiläufig gestrcift. Da die Normbereichselemente in der Ordnung der Konkretisierungselemente unmirelbar hincer den canones und noch vor Dogmatik- und Theorieelementen rangieren, wäre die Folge einer solchen Arbeitsteilung facal. Die Rechtswissenschaft könnte über die Delegavion ihrer zentralen Probleme an die Sozialwissenschaften sich als unzuständig für die Defizite ihrer Arbeitsweisen erkJären. Daß dies nicht beabsicheigt ist, ergibı sich aus dem Ansatz, eine nachpositivistische, soziale ReaLizäs einbeziehende, einheitliche Methodik der Konkretisierung zu enrwickeln. Sicherlich wäre es naiv, Müller vorzuwerfen, auf die zentralen Defizire der Rechtswissenschaft keine Anrwor parat zu haben, trorzdem hätte ich gewünscht, die Problematik wäre offener dargelegt worden. Denn die Frage, mir der ein Rezensent die Austührungen zur Normbereichsanalyse konfrontiert, trifft den wunden Punkr des Entwurfs. $W$ as z. B. soll als der Normbereich von An. 5 Abs. 3 GG ermizelt und geltend gemacht werden, von eincr Theorie der Instutution her die deutsche Universiüt in ihrer überkommenen Gestalt, von der Systemtbeorie her die Selbstdarstellung der Wissenschaftler oder von einer dialektisch-emanzipatorischen Theorie her die Herrschaftsfreiheit von Lehren und Lernen? Bedarf also die Ermitzlung und Geltendmachung des Normbereichs niche des theoretischen Zugriffs und darum der Entscheidung für einen theoretischen Ansarz? $\alpha^{9} \mathrm{Z}_{\text {war }}$ halte icb den Ruf nach einer Entscheidung zugunsten eines wissenschafustheorecischen Ansatzes in den Sozialwissenschafuen für überzogen, könnte die Jurisprudenz eine solche Ersscheidung doch nur in der Form einer Dezision fälen. Jedoch muß die juriscische Methodik fragmentarisch bleiben, solange die Rechrswissenschaft nicht in der Lage ist, zumindest Orientierungslinien für die im Normbereich zu verwendenden sozialwissenschaftlichen Techniken zu ziehen. Obwohl also bei der Normbereichsanalyse viele Fragen offen sind, ist das Schweigen über die

9 Berahard Schlirk. Jurisuische Mechodik zwischen Verizssungsthcorie und Wissenschaftstheorie, in: Rechischcorie, 7. Bd., (1976), S. 94 If., 10 . 
zu verwendende sozialwissenschafuliche $\mathrm{Me}_{\mathrm{e}}$ thode nicht zu verwechsein mit einer Resignacion des Juriszen engesichts der Vielfalt sozialwissenschaftlicher Theorien. Zunächst verhindert die Aufgliederung der Norm in Normtext und Normbereich die găngige Praxis, Realdaten in der Verkleidung normrextbezogener Gesichrspunkre unreflekriert in die Auslegung einzuführeo und die sprachliche Dehnbarkeit der Wortlaute zu überreizen. Sodann beansprucht das Posrulat der Methodenehrlichkeit Gelung auch fïr den Vorgang der Konkretisierung der Sachgesichispunkte, folglich wrird der entscheidende Jurist gezwrungen, bei der Betrachung und Verwernung van Normbereichselementen seine Wertungen offenzulegen und Auskunft zu geben über die angewandte sozialwissenschafuliche Technik.

Ernst Wolfgang Böckenförde sieht Müllers Ansacz in der hermeneutischen Tradicion der Freiburger Schule. ${ }^{\circ}$ Die Einordoung entspricbe niche dem wissenschafescheorecischen Selbstverständnis Müllers, sie ist auch zu ungenau, um den Arbeiren des Heidelberger Staatsrechtlers gerecht zu werden. Konkretisierung will Müllex nicht verstanden wissen als hermeneutische Ermitrlung des Sirnes einer substanzhaft vorgegebenen Norm, vielmehr volkiehs sie sich in einem Prozeß, in dem eine abstrakte Rechtsnorm in Beziehung gebracht wird mit einer konkreten Entscheidungsnorm durch Techniken meshodischer Zurechnung. (IV, 266) Juriscische Methodik umfaßt demnach $z$ was auch Hermeneucik, aber nur als eine methodische Technik unter anderen Konkrerisienungstechriken. Weil juristisches Arbeiten sich nicht in der Auslegung von Texten erschöpfu, also nicht auf Sprachlichkeir, Konmunikation und Verstehen reduzierbar ist, und weil die Verbindlichkeitsanforderungen in der Jurisprudenz ungleich höher sind als in den Geisteswissenschaften, kann und darl rechtswissenschafliche Methodik nicht aulgehen in geistes wissenschaftlicher Hermeneurik. Gänzlich verfehlt ist die Annahrne, Müller betreibe unter neuem Tizel das alte Geschäh topischer Rechestindung." $\mathrm{Zwar}$ arbeiter er in der Tat problem- und falbezogen, jedoch unter-

so Böckenlörde, Dic Methoden der Verkassungsinterprezacion - Besuandsaufnahme und Xrivik, NNW 1976, S. 2089 fl., 2096 f.

I Vermurungen in dieser Richtung bei Böckenförde, 2. 2. O., S. 2097. scheidet sich die juristische Methodik von topischen Ansätzen zentral durch die Funktion, die der Wortaut der Norm im Konkretisienungsprozeß einnimm?. Während für topisches Denken die Norm nicht mehr ist als ein Topos unter anderen Topoi, insistiern Müller auf dem Primat der Nombindung. Die Krivik an der Topik finder ihr Motiv niche primär in den Schranken der LeistungsJähigkeit topischen Denkens, sonders in der verfassungsrechtlich geforderen Bindung an den Normtext. Der Worlaut steckt die Grenzen verfassungsrechulich zulässiger Sinnvarianten ab. Damic ist zugleich die Prioritäı normtextbezogener Konkrecisierungselemente, vorrangig der grammatischen Auslegung, begründet. Problemlösungsstragegien, die diese Grenze überschreiten, mögen im Einzelfall methodisch vertretbar sein, verfassungsrechtlich zulässig sind sie nichr. Die Selbstbindung der juristischen Methodik zieht den scharfen Trennungsstrich zur Beliebigkeit topischen Argumentierens, die Grenzfunktion des Wortlauts ergibt sich aus wseinen normaciv geforderen Wirkungen für Rechessicherheit, Normklarheir, Publizitzät und für die Unverbrïchlichkeir der Verfassungsordnung im demokratischen Rechesstaate. (IV, ris)

Der wechselseitige Bezug von juristischer Methodik und Vertassungscheorie ist in dem Stellenwert, der den Grenztunkrionen des Wortlauts der Norm eingeräume wird, schon angcdeutet. Verfassungstheorie hat bei Müller eine doppelse Aurgabe, sic verarbeiter den Zusammenhang von Recht und Politik a und definiere die Grundbedingungen der juristischen Arbeirsweisen.

Die von Dubischar vorgetragene Kritik, das "Politische a flackere in der Methodik nnur ganz hincen am Horizonz als Fragesazz kurz auf ${ }^{1 x}$, überzeugr nicht. Vielmehr has Müller das Verhälenis seiner Methodik zur Politik in der Schrift "Juristische Mechodik und politisches Sysrem* eigens themacisien. Die Leugnung des Sachverhalus, daß juriscisches Arbeicen auch polivische Praxis isc, hält der Verfasser für "wissenschafilich sinnlos". Rechtsfunkrionäre sind keine "ungesellschaftlichen Glasperlenspieler ", die Forschungen zu Schichtzugehörigkeit, Vorverständnis etc. haben aufgeräumt mit der Ideologie einer "unpolitischen« Justiz und Rechtswissenschafr. Rückgebunden ist das Rechr an die

12 in: DOV 1973, 5. 289. 
Politik bercits im Entstehungsprozeß, wie überhaupt Recht sinsgesamt eine Sonderform von Policik und nach Voraussetzungen, Bedingungen, Funkuionen und Inhalten polirisch besuimmt (ist)*. (I, 44) Mit der Feststellung diescr Selbstverständlichkeizen ist jedoch nicht geklärn, wie mit diesem Tatbestand umzugehen ist. Eine Möglichkeit wäre, das Problem dahir aufzulösen, daß - wic es in einer gängigen Sprachformel heißt - malles politisch ist $\alpha$, und kritische Rechtsarbeit zu besummen als Ideologickritik. "' Demgegenüber insiscien Müller auf dem Sinn der Unterscheidung von Recht und Policik und der Typisierung ihrer formalen, normieren, insticutionalisierten Differenzen. (II, 49) Der rechtsstaatliche Anspruch, Recht und Politik durch verbindliche rechtliche Formulierung auseinanderhalten zu können, soll durch eine exakte juristische Mechodik beim Wort genommen werden. Dahinter steht die Auffassung, der graduelle Unterschied politischer Inhalte zu dem generellen Formalitätsvorsprung und Geliungsvorsprung gesetzter Normen sei wissenschaftlich faßbar. (II, so) Die Rechtsförmigkeir, in die politische Inhalte überführn werden, gestatcet die konstirucionelle Bändigung der im bürgerlichen Verfassungsscaat immer präseuten Gewalt.

Juriscische Mechodik erhält somit den Auftrag, mittels ihrer Rationalität eine sprachlich vermirtelbare Konfliktaustragung zu ermöglichen, d. h. den RückJall in eine Koppelung von Dezision und akrueller Gewalt zu verhindern. Dieser Rückfall ist deshalb immer möglich, weil Rationalität im modernen Ver\{assungsstaat zwei Pole umfaßr: Liberalisierung und Effektivierung von Herrschafu, Demokrasiechance und Herschafusfunktionalität. Die Zweideutigkcit bencrnt den Grund für die denkbarc policische Variante der Redukcion der demokrauschen und der Stäkung der autoritären Elernente im policischen

1) Die Beseimmung linker Recheskricit als ideologickricik lag nahe im CDU-Scast, als das Aufdecken des rabuisierten politisehen Gehalts von Recht und Rechuspraxis noch eine eminent polizische Sprengkraft zu entwickeln vermochic. Heute hingegen wird der fundamentalistisehon Formel vom Zusammenhang von Rocht und Politik mir Skepsis begegnal, verzichter doch die Reduktion von Rechrskrivik auf Ideologiekriok, die Rechespraxis an ihrem eigenen und normaiv geforderen Anspruch zu behafren, in ihurer Arbeit die Grundbedingungen für die Vorhersehbarkeit und Berechenbarkeit scaarlichen Handeins zu schaffen.
System der westlichen Industriestaaten. Juristische Mechodik ist solchen Involucionsprozessen nicht nonvendig hilflos ausgeliefer, vielmehr kann ihr eine retardierende Funktion in diesem Prozeß zukommen, indem sie nämlich das politische System zwingt, politische Liberalitäı rechtssuatlich korrekt zurïckzunebmen star bei unveränderter Normlage zu rechismethodisch nicht mehr belegbaren Dezisionen zu schreiten.

Mechodenklarheit und Methodenehrlichkeit erfahren ihre normacive Verankerung in den Formalgarantien und Formqualitäten des bürgerlichen Rechtsstaates, sie sind demnach keine voluntaristischen Postulate einer nur sich selbst verantwortichen Rechtswissenschafr und -praxis, sondern geforder vom posizuven Verfassungsrecht. Das Wissen um die treiheitsbedrohende Wirkung der Mißachtung der verfassungsrechrlich gebocenen Pulicht zu methodisch exakter Rechtsarbeit verleiht der Kritik an den Abwägungsenthusiasten des BVerfG unerbizdiche Schärfe."t Anders aber als Kriviker der staatsrechulichen Linken, die mit der Enclarvung der Praxis des BVerfG als Tradierung der 5932 von Carl Schmirt"s entworfenen Polivik des Gegeneinanderausspielens von Legiumität gegen Legalitär ihren Beitrag zur Verzeidigung formaler Rechusstaatlichkeit erbracht haben, verpflichtet Müllers Ansatz die Rechtsanwender auf die normativ unsermaverten Funkcionen, Strukturen und Arbeitsweisen des Rechtsstaaces. Dic Erhaltung des Verfassungsstaaces gegen Versuche, wesentliche rechisstaatliche Formalgarantien auf der Ebene der Rechespraxis herauszubrechen, ist getragen von dem politischen Credo, daß der Rechtsscaat nicht süberholt $\alpha$ ist durch Strukturveränderungen

14 Ein Beispicl: "Das Bundcsverfassungsgeriche is inzwischen offeabar gesonnen, nach der Abhör- und Hochschulenuscheidung aus/ dem Weg übcr Grundlagenvertrags-, Abreeibungsund Radikalenjudikat das Verlahren der me thodisch inkorrekren, rechulich dubiosen und sprachlich schwammigen Dezision zu cinem für die rechlsstaaticho Demokratie sinisacn Forzsczungsroman auszuweitcn.a (11, S. 27)

is Vgi. die in Sommar 1932 zum Zwecke der stanesrechrlichen Vercidigung des Präsidialkabiners Papens geschricbenc Schrift Sehmites, Legalität und Legitimitàt, wiederabgedr, in: đers., Verfassungsrechrlichı Aufsātze, Berlin 1973, S. 263 H. Die Akcualität des Probiems einer azueiscufigen Legalitär a fü das polivisehe System der Bundesrcpublik hat dergelegr erstmals Preuß, Legalitït und Pluralismus, FrankfurdMain 1973. 
in politisch-ökonomischen System. Selbst wenn die akruell kaum verifizierbare These zucriff, daß zwischen den normativen Strukturen und dem politischen System keine funktional hinreichende Übereinstimmung mehr besteht, ist mit den Ergebnissen dieser Analyse keineswegs die Gegenstrategie bestimmt. Preuß hat eine mögliche Strategie skjzzier, wenn er die Entracionalisierung des Rechessystems als unverrïckbares Fakrum hinnimmt und zu einer Rechtsstaatskunde des Nllags", die Widersprüche, Friktionen und Interessengegensatze innerhalb der herrschenden Oligarchien auszunutzen weiß, rät. ${ }^{16}$ Müller hingegen begreift die Vereidigung formaler Rechusstatlichkeit als Aufgabe politischer Praxis auch der Juristen, die beraten werdea, in ihren alltäglichen Arbeitsweisen den rechtsstaatlich geforderten Nachweis der methodisch korekten Rückführung konkreter Entscheidung auf Rechtsnormen zu erbringen und norfalls das politische System zum autorizären Olfenbarungseid zu zwingen, ansur dem schleichenden Übergang in eine nachliberale Suarsform der "modernen Industriegesellsehafta juristische Schüizenhille zu Jeisten.

Die Arbeiten Friedrich Müllers sind von der staatsrechulichen Linken nicht rezipiern worden. Dies ist erstaunlich, weil Müllers Schriften die zentralen Themen der in der westdeutschen Linken geführten statsrechtlichen Diskussion wie Entformalisierung des Rechts, Rechtsstaat und Gewalt, Sozialwissenscbafien und Jurisprudenz zum Gegenstand haben. Der Grund für die ausgebliebene Rezeprion könnce in der Behandlung der genannten Themen zu finden sein. Müller krivisiert die Defizite der Rechtswissenschaft aus der juristischen Perspektive, während dic kritische Staatsrechtsliteratus ansonsten dazo neigr, von den Sozialwissenschafien aus die Defizite der Recheswissenschaft anzugehen. So erarbeiter Müller beispielsweise seinen mechodischen Enrwurt in der Auseinandersetzung mit der juristischen Methodendiskussion. Dieser Sachverhalc könnte vielleichr erklären, warum gegen seine Publikationen

16 Preuß, Nachuräge zur Thoonie des Reclus. stasts, in: Tohidipur (Hrsg.), Der bürgerliche Recherstzat, I. Bd.. Frantelure/Main 1978, S. 82 ff., 98. Deutliche Abstriche an diesem Konzepe macht PreuB híngeger in dem Aufsalz - Lckalič̈́ - Loyalizät - Legitimitätu, in: Leviathan, 4/77. S. 450 ff. der Einoand der Unverständlichkeiz erhoben wird. Dem sozialwissenschaftich orienvierten Leser mag eine juristische Sprache, die sich selbst an dem methodischen Anspruch auf Klarheit behaften läßr, schwer verständlich erscheinen, der Hinweis aul die Angemessenheir von Sprache und zu behandeindern Problem gilt jedoch auch hier. Bedauerlich wäre die Ignorienng dieses Ansalzes, da die Einheit von Verfassungstheorie und juristischer Methodik für kritische Juristen und insbesondere Rechtspraktiker eine politische Handlungsperspekuive eröffnen könnce. Die Praxis linker Anwälre etwa ist vielfach gekennzeichner durch einen recht dezisionistischen Umgang mit juristischen Methoden, einmal wird strikt positivistisch argumentierr, ein andermal die Topik bemüht und, wenn es denr sein muß, werden auch materiale Argumente vorgetragen. Zusammengehalten werden die verschiedenen Strategien allein in der Person des Rechespraknikers. Diese Form der s Rechessraatskunde des Allrags $\propto$ lebı von der zufälligen Verbindung ciner guten, d. h. hier: linken Gesinnung mit beliebig handhabbaren und einsetzbaren juristischen Techniken. Müllers Ansatz hingegen reflekcier die Methoden der Rechisanwender im Kontext einer Verfassungscheorie, welche dic Oberprüfbarkeit, Diskutierbarkeit und Regularität juristischer Entscheidungsfindung festmacht an den Strukcuren des liberalên Verfassungsstaates. Uber die damit angezeigre Option muß man sich freilich im $\mathrm{kla}$ ren sein: es geht um die Erhaltung und Entfaltung der politischeo Emanzipationschancen des bürgerlichen Rechrsstaares.

Von den rechismethodischen und verfassungstheoretischen Arbeiten Müllers kann auch eine kritische Rechiswissenschaft lernen. Die neuerdings auch von ihr beschworene OHenheit der Verfassung « ist gegen materiale Verkürzungen nicht dadurch gefeit, daß die Terminologie gewechselt und scatt von Offeaheit von cinem wstruknurierten Forum gesprochen wird. Seiferts Kritik an der Umwandlung des Forums in eine Wertekirchca muß solange in einem schlechten Sinne abstraku bleiben, wie den Wercphilosophen des BVerfG lediglich eine Gemengelage von polizologischen Exorzismen und moralischen Appellen entgegengesetzt wird. Wenn die staatsrecheliche Linke über den Verfassungspositivismus binausgelangen sill, wozu Seiferr räı, kann sie nicht stehenbleiben bei dem Plädoyer zur $\star$ Rückkehr zum liberalen Ver- 
fassungsstaat $\alpha$, sondern wird Auskunft geben müssen, wie ihr verfassungstheoretischer Ansatz sich verhält zum positiven Verfassungsrecht und welche juristischen Mechodiken geeignet sind, die Rechtspraxis zurückzuzwingen in die rechtsstaatiche Bindung an das posirive Recht, d. h. sie wird den dürren Boden der juristischen Methodendiskussion bearbeiten müssen. Müllers Arbeizen geben eine Ebene der Fragestellung vor, unterhalb derer die Herstellung einer Verbindung von Rechtsmethodik und Verfassungstheorie Flucht in einfache Theorie wäre. Der $\gg$ Kampl um Verfassungspositionen « ist zu ergänzen um die Arbeit an ciner juristischen Meshodik, Müller hat hicrus wichwige Vorarbeiten geleistet.

Volker Neumann

Poluische Studien, Sonderheft $2 / 1979$, Grundgesetz und politische Wirklicbkeit. München, Günter Olzog Verlag, 7,80 DM, (1.).

Josef Isensee, -Verfassung ohne Emstfall: der Reclusstaalx, in: Anton Peisl u. Armin Mohler, Hrsg., Der Emstfall, Schriften der Carl Friedrich von Siemens Stiftung, Bd. 2, Frankfurt/M.-Berlin-Wien, 1979, Verlag Ullstein, 29,80 DM (II.).

-Linke Juristen haben in den vergangenen Jahren wiederholt die Erosion des Grundgesetzes kritisiert und in dieser oder jener Form die Aushöhlung der Verfassung durch Interpretation des Grundgeserzes als Wertsystem, durch Erhöhung der ofdGO zu einer Oberverfassung, durch vorverlegten Staassschutz oder übergesetzliche Norstandsrechte analysiert. In einem aus Anlaß des 30jâhrigen Bestehens der Bundesrepublik von der Hanns-Seidel-Stikung (CSU) in München veranstalteten Symposion drehen sich ein Teil der Referate um das Stichwor "Vertassungsverbiegung a'. Auf den ersten Blick sieht es so

\footnotetext{
1 Die von konservariven Suitungen finanzieren Vorkaben solleca im Zusammenhang gosebcs werden: Der Hanns Sendel-Sritung ging es ig79 um - Verlassungsverbic gung : ic dic Carl Friedrich Siemens Srifuog veranstaltete im Juni und Juli $\times 97^{8}$ eine Voreragseitic zum Thema -Der Ermsifall. und dic Thyssem-Srifung unierstizi rinen Arbeitskreis über den Begrith > Regietbarkeit (siche dazu dic Racasion von Dreier / Uthmann in: KJ 1980, S. $2: 6$ if. .
}

aus, als ob die linke Kritik an der Verfassungsencwicklung der Bundesrepublik aufgenommen wird vom konservativen Lager. Doch der Schein trüg.

Das Stichwor *Verfassungsverbiegung " gibr Franz Joseł Strauß (I, S. I 8) in seinem Beitrag "Das Grundgeserz aus der Siche des Politikers*. Strauß spricht von weiner verbogenen oder verschobenen Auffassung über Sinn und Zweck des Grundgeserzes*, läßr es im übrigen aber offen, wob am Kembestand der grundgesetzlich garantierren Rechte und Frciheiten schon genagt wird oder riche (S. 13 f.). Gemeint ist die SPD-FDP-Regierung in Bonn. In dicsem Sinn wirf auch der CSUGeneralsekretär Edmund Stoiber der SPD weine Abkehr von einem Verfassungsverständnis* vor $\langle S .7\rangle$, was vermutlich heißen soll, einc Abwendung vom Verfassungsverständris der CDU und CSU.

Walter Leisner, Ordinarius für ölfontliches Recht an der Universität Erlangen, untermauen diese Thesen mir cinem Referat $*$ Die Verfassungsverbicgung $\alpha$. Leisners These: "Vor der Verfassung stehe das Bundesverfassungsgericht mit dem flammenden Schwert: Der offene, gezielte Verlassungsbruch ist unmöglich. Was also bleibr ist ... die Verfassungsverbicgung * (S. 57). Er nennt die Verfassungsverbiegung eine "Strategie der ¿Mehrheit des Bundestages $\alpha$. Die $\backsim$ Werte des Grundgeserzes" würden dabei *niche geleugnet , sondem lausstark sverkündet «. Die Verfassungsverbiegung erfolge เoninuierlich und als, Verlassungsverletzung in vielen kleinen Schritten, ... stetes Gesetz höhlt die Verfassung $\alpha$. Die Grundregel jedes Verfassungsverbiegens (nwie jedes Biegens $\alpha$ ) sei: - Immer bis an die Grenze gehen, ... es darf nicht zum Verfassungsbruch kommen* (S. $s_{7} \mathrm{H.}$.). An anderer Stelle räumt Leisner ein, "Verfassungsverbiegung ist eine Versuchung jeder Regierungsmehrheic* (S. 62). Doch die gegenwärige Bonner Regierungsmehrheit sei dieser Versuchung win besonderem Maß a ausgelieferz: Denn diese Koalicion halse die Ideologie, also wein großes fernes Ziele, auf das shingebogen $\alpha$ würde; sie haben die wklare, ständig gehaltene Richrung*, wden Willen, an die Grenzen zu gehen $\alpha$ und verfüge über eine gleichbleibende Maclutkonstellation, über Konuinuität (S. 62 f.).

Neben Leisner scellt sich Walter Schmitt Glaeser, Ordinarius für ölfentliches Recht an der Universirät Bayrcuzh. Unter der niche gerade neuen Uberschrife nVerfassurig und 
Verfassungsreche untersuch er "Verfas sungsversiöße von oben " und beklagr ein „Dezennium der Verfassungsverstöße $\alpha$, das hintar uns licge (S. 37).

Woran machen die beiden Staatsrechislehrer "Verfassungsverbiegungen a und "Verfassungsverstöße von oben $\propto$ lest? Leisner nennt als Beispiele: $"$ Die zahliosen Versuche, im Namen übersteigener sog. Sozialbindung das Eigenturn dergestalt zu enrwerten, daß es sozusagen in den Händen der Eigentümer enteignet wird", die "Verbiegungsversuche des Eigentums im Bodenreche und in der Mitbestimmung "und die "Aushöhlung des Berufsbeamtentums ("geradezu ein klassisches Beispiel der Verlassungsverbiegung ). Beim Beamicntum war man, so Leisner, s schon süber der Grenzc « ; doch das Bundesverfassungsgeriche habe nnoch einmal zurückgebogen und die sesondere Treuec verlangt * (5. 59-62). Für Schmiu Glaeser ist die Fristenregelung sogar ein nqualifiziercer" Verfassungsverstoß, nder aul den Nerve dcs Grund gesetzes gerichtet war a (S. 41). Die von der Bonner Koalirion beschlossene Neuregelung des Anerkennungsverfahrens für Kriegsdienstverweigerer habe die "Funkuionstähigkeit « der Bundeswehr gefährdet (S. 43 ff.); auch die "Abschaffung der sog. Regelaníragen bei den zuständigen Verfassungsschuczämterna sei ein Verfassungsverstoß (5. 47 f.), die Neuregelung des elterlichen Sorgerechces führc - man höre! - zur "Zerstörung « oder "Verstaatlichunge der Familie (S. (3).

Bezeichnend für den Scil ist, daß beide Staatsrechtler solche Vorwürfe zwar erheben, daß sie aber - sofern sie nicht auf die einschlägigen Entscheidungen des Bundesverfassungsgerichtes verweisen können - gar nicht erst versuchen, die „Verfassungsverbiegungen a oder -versë̈ße $\alpha$ durch eine an den Normen des Grundgeserzes orienticrte Verfassungsauslegung zu belegen. Bereits diese Tatsache muß als Eingeständnis für die argumencarive Schwäche der vorgetragenen Position angesehen werden (was nicht dazu führen darf, tassächliche Machepositionen und Einwirkungsmöglichkeiten zu unterschätzen). Leisner geht noch weiter, wern er sich ausdrücklich gegen stechnische Pragmatik « ausspricht und sagh nnur von der hoben Warte des Prinzipiellen, der Verfassungswere aus wird die Verlassungsverbicgung überhaupt sichtbar $\alpha$ (S. 63).

Um welche "Werten es dabei gehc, wird deurlich, wenn sich Leisner gegen die "offene
Verfassung $u$, sogar gegen die Formel einer Nichufestlegung des Grundgesetzes auf einc - Wirtschaftsverfassung a und die darin enchaltene sWerneutralizäta des Grundgesetzes ausspricht und Begriffe, die das Bundesverfassungsgericht im Mitbestimmungsurzeil inzwischen emeut bestätigc hat, dem $n$ Auflösungsprozeßa der Verfassung zuorónet. Damic zeigt er, daß diese Position mit der an den Normen des Grundgescrzes orientieren Kritik der "Erosion der Verfassung* niches gemein hat. Es ist nicht zufällig, daß diese Kritik nicht einmal erwähnt wird. Leisner und Schmitr Glaeser lasten ebenso wic Franz Josef Scrauß der SPD als Verfassungsverbiegung oder Verfassungsverstoß ein politisches Verhaiten an, das sie selbst betreiben.

Einen anderen Weg als solche Gleichserzung von Verlassungsrecht mit den eigenen policischen Werten weist Josef Isensee. Auch or meint: ${ }^{\mathrm{D}} \mathrm{Da}$ die Verfassung als Basis der politischen Einheit akzepcien ist, biezer sich Verlassungsauslegung als Hebel an, den Grundkonsens zu bewegen und die Umwerrung der Werte einzuleitena (II, S. I 18). Ohne Beleg und ohne Blick für die überwiegend konservativen deusschen Staatsrechtslehrer behauptet Isensee, Ordinarius für öffentliches Recht in Bonn, die "Erben der deutschen Kulzurrevolucion" hätten diesen Hebel längst entdeckr $*$ : "Die dem Scaat des Grundgeseczes gemäße Form der Revolution isc die Subversion des Verfassungsverständnisses. Revolution erfolgt heute durch Verfassungsinterprerasion" (ebd.).

In Unterschied zu Leisner und Schmin Glaeser untersucht Isensee zwei Beispiele, in denen wrechisstaacliche Selbstbindung" nach dem Grundgeserz durch "gesetzesfreic Abwägung ersetzı worden scien: die SchleyerEntfuihrung und die Abhör-Alfáre Traube: wIn beiden Ereignissen, im echten Ernstfall und im vermeindichem Emstall, flammte das archaische Norreche des Sizates auf. In der Grenzlage der woulen Herausforderung zerriß die Starsgewalt das verfeinerte, elfizienzhemmende Geflechr der Legalizär - unter Berufung avf die Legitimicät. Der formalisierte Gesetzesvollzug aurde erseczt burch gesetzesfreie Abwägung vertassungsgeschüuzter Gürer « (S. $1 I_{3}$ f.).

Doch Isensee spriche nicht von "Verfassungsversto $\beta_{*}$, auch nicht von einer ${ }^{\prime} K r i s e$ des Rechisstaates schlechathin $\alpha$. Er wendet sich gegen die "Hypertrophie des rechtscaarlichen Bestimmtheitsgebotes und gegen das 
"Ubbermaß staatlicher Selbstbindung" (S. I I 4 u. r17). Um das zu sichern, was Isensee * staatliche Selbstbehauptung $\alpha$ nennt, plädiert er im Verfassungstecht für den wheute fast susgestorbenen Regelungstyp der Generalklausel, wie sie das preußische Polizeireche entwickelt hat $\alpha$, mit anderen Worten für die Einfübrung der 1968 verhindernen Generalklausel im Notstandsrecht (S. I I4).

Isensee räumt ein, daß sich staadiches Handeln außerhalb der Verfassungsiegalicät vollzogen hat ("Legal waren diese Maßnahmen nicht $\alpha$, S. I 2 ); cr legitimicr mit Hilfe dieser von ihm (in der Sache, niche in der Begründung) colerierten Verstöße gegen Legalität den Ruf nach Verfassungsänderung und fordert Ausnahmebefugnisse für die Exekuive! Das weffizienz-hemmende Gefleche der Legalizäs« will er nach dem Muster von ArT. $4^{8}$ Weimarer Reichsverfassung und unter Berufung auf die Argumencationslinien der CarlSchmir-Schule lockern: Wenr es nicht daraut ankommt (in der Normallage) "grundrechtssichernde Staatsbegrenzung $\alpha$, in der Ausnahmesituation Vertassungs-sElasuizitïra zu Gunsten der Exekutive. - Leisner und Schmitt Glaeser dagegen betreiben Umwertung der Were. Die hohe Warte der Verfassungswerte erweist sich bci ihnen als der Kirchrurm der CSU.

Beide Positionen repräsentienen unterschiedliche konservauive Ansätze, den durch die Normen des Grundgeserz geserzten Rahmen zu sprengen. Bei Leisner und Schmitc Glaeser wird der gesellschaftliche status quo zum Verfassungswert erhoben, insbesondere die bestehende Wiruschaftesordnung mit Verfassungsrecht idencifizier; bei Isensee soll die Exekutive (unabhängig von einer CDU/ CSU-oder einer SPD/FDP-Regierung) in die Lage versetzi werden, gegen eine Bedrohung ökonomischer und sozialer Besirzverhältnisse vargehen zu können. Beide Ansätze - sowohl die Marerialisierung der Verfassung als auch die Suspendierung von Verfassungsrechten dienen demselben Ziel: der Fescigung und Aufrechterhalung von Herrschaftsverhältnissen.

Jeder Ansarz benörigr zur Durchserzung dieses Zieles ein spezielles Feindbild. So heißt es bei Lcisner: "Die Radikal-Demokratisierung ist der Vernichrungsangriff auf das Grundgesetz* (Y., S. ऽ6); Ysensee dagegen formulier: - Die rationale Revolutionstechnik ist die Legalität. Der Scaar des Grundgesetzes hat nicht den Barrikadenkampl zu fürchten, son- derm den Marsch durch die Instirutionen « (II., S. IIs). Sind solche Feindbescrmmungen nich Wegweiser in eine anderc Republik, in der radikale Demokraten dann Staatsfeinde sind und in der das Unterfangen, odie rechrstaaclichen Garancien der Legaliät und der Grundrechte zu akuivieren «, bereirs ein Arhalspunkt für die Bestimmung des Verfassungsgegners ist?

Jürgen Seifer

Helmut Sclsulze-fielizz: Sozialplanung im Städubaurecht, Atbenäum Verlag, Kronberg i. Ts, 1979. 458 Seiten, $D M$ 130,-.

Wie der bekannte Kritiker des BGB, Orro Gierke, die wenigen Schutzvorschriften für im Rechesverkehr benachceiligte Gruppen spöttisch als xden Tropfen sozialen Ols, mit dem das Gesetz gesalbt sei ${ }^{\prime}$ bezeichnete, so haben Sozialwissenschafiler die Bestimmungen des Städtebaufördenungsgesetzes zur Sozialplanung schnell als bloßses „Schmiermirtel* zur schnellen Abwicklung von Scadcerneuerungsmaßnahmen abqualifizier. Nur selten ist der Versuch untemommen worden auszuloren, welche Chancen catsächlich gegeben sind, mil Hilfe dieser Bestimmungen negarive Auswirkungen solcher Maßnalymen zu vermeiden.

Nun haben in den letzter Jahren Soziologen die Probleme der Sanierung und der Sozialplanung aufgegriffen, durchaus um die praktische Verwertbarkeit ihrer Arbeiten bemüht. Planer können heute, anders als zum Zeitpunkt der Verabschiedung des Städtebauförderungsgesetzes, auf eine Fülle von prakuikablen Forschungsergebnissen' zurückgreilen. Es fehlte aber bisher, und hier ist

\footnotetext{
2 So heißs as bei Isensec (Il., S. 116 f.): „Der Verlassungsgegner bemüht sich, dic rechisstaallichen Garantien der Legalicär und der Grundrechre zu akeivieren, um den Zugang zum öfentlichen Aorı zu erzwingen.* Die große Gefahr sieht Isensee in der $n$ Rückkchr zum Weimarer System der Icgitimin̈üsfreien Legalieäta (S. 117). Dabei legt er - wic Carl Schmirt 1932 den Weg Irei für cinc Icgalititssfreie Legiumitäe. I Vgl. F. Wicacker, Privaurechugeschichue der Neuzeit, 2. Avfl, Göringen 1967, S. 470.

1 Zusammenfassend: U. Herlyn, Sozialplanung und Stadrerneverung, Sturgar 1976.
} 
$34^{8} \quad$ es Schulze-Fielirz gelungen, eine Lücke zu schließen, an sozialwissenschafelich orientierten Beurteilungs- und Bewercungskriterien für Juristen, die ja ebenfalls, was von Sozialwissenschaflem viel zu wenig bcachter wird, gestaltead in die Sozialplanungsverfabren eingreifen. Wer für die Lösung rechelicher Probleme, z. B. im Zuge von Sanierungen, auch sozialwissenschaftliche Erkenntnisse heranziehen wollue, fand in der soziologischen Litcratur kaum verwernbare Ausführungen. Insorem ist die Doppelqualifikation als Rechrswissenschahter und Sozialwissenschaftler, die der Autor besizz, bedeursam. In einem einführenden Kapitel wird zunächst die Problcmatik von Stadterneuenungsmaßnahmen historisch angegangen, wobei der Akzent auf dic Wohnungs- und Südtebaupolitik scil 1945 gelegi wird. Zu Recht werden die unter der Utberschrift, Krise der Stadı diskuvierten Einzelfragen, wie die Defizite an sozialer In/rastrukrur, der Verlust lokaler Kommunikation, dic Entstehung von Sanierungsgebieten, um nur einigc Beispiele zu benennen, als Folgeprobleme einer wstädtebaulich, vereilungs- und sozialpolitisch bedenklichen liberalisienen Wohnungsmarkspolitik (S. j92) begriffen. In der zunehmenden Beachrung der Sozialplanung im Stadrebaurecht, ablesbar an den gesetzgeberischen Aktivitäten der letzten 10 Jahre, wird aber ein Ansarz zur stadtplanungspolitischen Korrektur gesehen.

Damit ist der Gegenseand der Untersuchung benannt, aber noch nicht besuimmt. Aus der breiten, terminologisch und sachlich ungeKärten Diskussiona (S. 393) innerhalb der Sozialwissenschaften, was denn nun eigentlich unter dem Begriff Sozialolanung zu verstehen sei, lassen sich seiner Meinung nach keine brauchbaren Abgrenzungskriterien entwickeln, stan dessen greift er auf die gesetzlichen Bestimmungen zurück, nach denen sich unterscheiden lassen:

- die Sozialplanung im engeren Sinne als Hilfe und prävenxive Rücksichtsnahme bei Sanierungen und Gebiersumgestaltungen nach $\$ \$_{4}$ II, 8 II StBauFG und $\$$ I 3 a BBauG - die in ihren sozialen Gehalten sensibilisieric Sanierungs- und Bebauungsplanung nach $\$ 1,3,4$ I 86 I StBauFG und $\$ 1,2$ ff. 9 a BBauG

- und schließlich die parriziparive Ausgestal. tung des Verfahrens dieser bciden Elemente. Schwerpunkt der Arbeit sind die ersten beiden Fragenkomplexe. In ejnem besonderen
Kapirel, das vorwiegend den an Rechtstheorie Interessierten zur Lekrüre empiohlen werden kann, versuche Schuleze-Fielitz seine These zu belcgen, daß Stadrerneuerung als Sozialplanung grundgesetzlich autgegeben sei. Nicht jeder wird seiner Ansicht folgen und das teilhaberechuliche Grundrechesversündris als Voraussezzung für eine dogmausch befriedigende Beriicksichtigung des Sozialstaassgrundsaczes begreifen. Trotzdern, es wird kennunisreich belcgt, daß von der Verfzssung her dic Möglichkeit gegeben ist, die Sozialplanung in ihrer konkreten Austormung in Städrebaurecht als Aułgabe des Gundrechtsschutzes aufzufassen.

Die Vorbehalte von jurisischen Prakcikern gegenüber der Soziologie sind der Anlaß für längere, ein ganzes Kapitel einnehmende Ausfïhrungen über das Verhälınis von Rechtswissenschaften und Sozialwissenschatten. Dabei wird deuttich: eine Sozialplanung ohne Rückgriff aul die Ergebnisse der Stadtsoziologie wird dem geserzlichen Auftrag nicht gerecht. Im $\$_{4}$ StBauFG wird sogar eine an der empirisch-analyrischen Forschungslogik orientiene sozialwissenschaffliche Sanierungsvorbereitung ausdrücklich vorgeschrieben. Auch die in $\$ 3 \mathrm{SzBauFG}$ geforderte Beurzeilung der baulichen und sozialen Verhälenisse eines Sanierungsgebietes ist nur innerhalb eines Theorierahmens der Stadcsoziologie möglich. In mehreren Kapiceln werden dann in Anlehnung an den cassächlichen Verlauf von Scadeerneverungsmaßnahmen, von der ersten anaiyrischen Bescandsaufnahme im Rahmen der vorbereitenden Untersuchungen bis zur Aufstellung und Durchfïhrung des Sozialplans, dic sozialplanungsrelevanten Besummungen kenntnisreich mit den Ergebnissen soziologischer Forschung ausgefüllt.

Auch wenn die Planung der Durchfïhrungsfolgen in der Praxis bisher leider nicht den angemessenen Stellenwer erhalten hat, so ist diese als ndas begriffsprägende Kernstück der Sozialplanung $\alpha$ (S. 401 ) anzusehen.

Als eigentlicher Fix- und Angelpunki dieser Planung sind die Grundsätze für den Sozialplan nach $\$ 4$ II y SuBauFG bzw. die Grundsätze für soziale Maßnahmen nach $\$ 1\}$ a I BBau $G$ anzusehen. Nur wenn sie, rvas bisher allerdings kaum geschehen ist, genau, derailliert, informativ und adressatengerechr formuliert und von der kommunalen Verretung beschlossen werden, kann der cinzelne sein "Planungsschicksal $\propto$ kalkulieren. 
Die Widerstände gegen eine stárkere Berücksichuigung der Sozialplanung im Prozeß der Stadterneuerung, die zumeist eine Erhöhung der Kosten und eine zeisliche Verzögenung bedeuret, werden von Schulze-Fielizz gesehen. Trotzdern sieht er Chancen, mir dem Vchikel der Sozialplanung das ngesamte Handeln der Verwaltung sozial zu sensibilisieren (S. 406), eine Holfnung, die durch die bisherige Praxis von Stadterneuerungsmaß nahmen nur wenig gesrützt werden kann.

Obwohl überwiegend aul den Gebrauch durch prakrisctı tätige Juristen abgestimmt und deshalb wie ein Nachschlagewerk aufgebaut, bietet die Arbeit auch einen vollständigen Uberblick über Forschungsergebnisse (und -lücken!) des Sozialplanungsprobleme themarisierenden Bereiches der Stadtsoziologie und ist insofern auch für den Sozialwissenschartler ein gut brauchbares Fundstellenverzeichnis.

Es gelingt Schulze-Fielizz mit juristischen Cberlegungen die Notwendigkeir einer Aufwertung der Sozialplanung gegenüber der Bauplanung zu begründen und damic richtig zu gewichten. Dic rechtliche Absicherung der Sozialplanung ist, und darin ist dem Verfasser zu folgen, auch wenn Grundgedanken des Städrebautörderungsgeserzes bei Gelegenheit der Novellierung Eingang in das Bundesbaugcserz gefunden haben, noch immer unbefriedigend.

Das gegenwärtige Boden- und Planungsrecht wird von ihm treffend als krafulos bezeichnet, was in der Praxis dazu führi, daß auch bei einer konsequenten Answendung der einschlägigen Bescimmungen durch Verwalcungen und Gerichte eine Bauplanung, die auch die Bedürfnissc und Interessen van sozial Schwächeren (besonders Mietern) berücksichtigt, nur schwer zu realisieren ist, obwohJ niche verkannt wird, daß sich manche sozialplanerischen Grundsäıze durch eine differenziene Ausschöptung des gelrenden unmitrelbar oder mictelbar einschlägigen Landesoder Bundesrechts verwirklichen lassen.

Die gefordere Verbesserung der Entschädigungsregeln für wirtschafilich Schwächere könnre die negativen Auswirkungen des sbedenklich liberalisierten * (S. 392) Wohnungsmarkies wolsl mildern helfen. Es ist jedoch zu befürchten, daß die geringe Finanzkerafe der Gemeinden zu einer noch restrikuveren Handhabung solcher Bescimmungen führr. Auch bei einer Verbesserung ihrer rechtlichen Stellung müssen sich deshalb z. B. Sanierungsbetroffene weirerhin auf

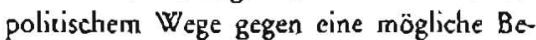
drohung ibres sozialen Besizzstandes wehren. Ohne die praktischen Probleme beim Umgang mit sozialwissenschafilichen Arbeiten in der juriscischen Praxis zu untcrschätzen, siehe Schulze-Fielitz besonders in den cmpirisch-analytisch ausgerichueten Forschungen wichuige Entscheidungshilfen.

Durch den behursamen Umgang mit rechrswissenschafelichen und sozialwissenschaftlichen Theorien, Methoden und Begriffen wird für cine längst fällige Annäherung der Disziplinen geworben und überzeugend nachgewiesen, $d_{2} ß$ dic bisherige Gewohnheit, eher aut Abgrenzung zu anderen Wissenschaften zu bestehen, der komplexen Problemacik der Sozialplanung niche gereche wird.

Günter Wends 